\title{
FITOQUÍMICA E ATIVIDADE FARMACOLÓGICA PRELIMINAR DO EXTRATO AQUOSO DE Tynanthus micranthus Corr. Méllo ex K. Schum: UM EXEMPLO DA CAPACIDADE PREDITIVA E DOS CUSTOS NO DESENVOLVIMENTO DE NOVOS PRODUTOS FARMACÊUTICOS
}

\author{
Cristiane Loiva Reichert¹, Ana Paula R. Costaํ, Andressa Gazola1', Claudini H. Di Pieri', \\ Evelyn C.S. Silva', Thereza C. M. de Lima ${ }^{1}$
}

\author{
1 UFSC \\ Correspondência: Thereza C. M. de Lima: thereza.lima@ufsc.br
}

\begin{abstract}
RESUMO: Os produtos naturais têm servido de inspiração para o desenvolvimento de vários produtos pela indústria farmacêutica local e mundial. O objetivo do presente trabalho foi avaliar a atividade farmacológica preliminar do extrato aquoso das folhas secas de Tynanthus micranthus, usando o teste hipocrático em camundongos, como uma ferramenta para direcionar estudos farmacológicos futuros, bem como avaliar os custos envolvidos na execução deste estudo. A T. micranthus Corr. Méllo ex K. Schum, conhecida popularmente como cipó-cravo, é facilmente encontrada na região Noroeste do Estado do Paraná, Brasil, sendo utilizada pela população como calmante e analgésica. No entanto, existem poucos trabalhos científicos com esta espécie vegetal. $O$ cálculo do custo contábil foi realizado para simular o valor gasto por uma empresa privada na realização destas atividades de Pesquisa e Desenvolvimento. Nossos resultados mostram que além do baixo custo, o teste hipocrático proporciona resultados importantes para a decisão da via de administração, toxicidade aguda, efeitos com altas doses e por diferentes vias, além de fornecer informações sobre a latência para os efeitos observados. Com essas informações e uma análise fitoquímica mais aprofundada, isolando e identificando os compostos majoritários, é possível avaliar na literatura a relação composto $x$ atividade farmacológica direcionando de forma mais assertiva as pesquisas a serem realizadas. De maneira geral, apresentamos dados inéditos da espécie estudada ( $T$. micranthus), mostrando uma estratégia de triagem farmacológica simples e de baixo custo para a pesquisa de plantas medicinais nativas que apresentem informações restritas na literatura e que possam servir para o desenvolvimento de novos produtos farmacêuticos.
\end{abstract}

Palavras-chave: Bignoniaceae; plantas medicinais; teste hipocrático

\section{PRELIMINAR PHYTOCHEMISTRY AND PHARMACOLOGICAL ACTIVITY OF THE AQUEOUS EXTRACT FROM Tynanthus micranthus CORR. MELLO EX K. Schum: AN EXAMPLE OF THE PREDICITIVE AND COSTS VALUES IN THE DEVELOPMENT OF NEW PHARMACEUTICAL PRODUCTS}

\begin{abstract}
Natural products have been an inspiration for developing several pharmaceutical products by the local and world industry. The aim of the present study was to evaluate the preliminar pharmacological activity of the aqueous extract from leaves of Tynanthus micranthus, using the hippocratic test in mice as a tool to guide the pharmacological studies, as well as to estimate the costs involved in this process. T. micranthus Corr. Méllo ex K. Schum, popularly known as cipó-cravo, is easily found in the Northwest region of the State of Paraná, Brazil, being used as tranquilizer and analgesic folk medicine. However, there are just few scientific studies on this plant species. The evaluation of financial costs was performed to simulate the value spent by a private company in the realization of these Research \& Development activities. Our results show that the Hippocratic test is a low cost test, and produces important results to take the decision about the route of administration, the acute toxicity, pharmacological effects, giving information about the latency to observe the effects. This information plus a further phytochemical analysis, isolating and identifying the major constituents, besides the evaluation in the literature about the relationship between compounds $X$ pharmacological activities, guiding the research to be done. Overall, In the present study we presented an unpublished pharmacological activity (analgesic) for T. micranthus, showing a strategy of a simple and of low cost pharmacological screening to the research of native medicinal plants that present few information in the literature and that could be useful for the development of new pharmaceutical products.
\end{abstract}

Key Words: Bignoniaceae; hippocratic test; medicinal plants 
Fitoquímica e atividade farmacológica preliminar do extrato aquoso de Tynanthus micranthus corr. Méllo ex k. Schum: um exemplo da capacidade preditiva e dos custos no desenvolvimento de novos produtos farmacêuticos

\section{INTRODUÇÃO}

Os produtos naturais tem servido de inspiração para o desenvolvimento de vários produtos pela química orgânica nos últimos tempos. A avaliação de extratos e de compostos derivados de plantas servem como um caminho para o avanço de metodologias de síntese e a possibilidade de fabricação de análogos do composto original, melhorando seus aspectos farmacológicos e farmacocinéticos (Harvey, 2008), buscando sempre uma maior eficácia e segurança com menos efeitos adversos. Atualmente, os produtos naturais e seus derivados representam mais de $50 \%$ de todos os fármacos em uso na clínica médica (Gurib-Fakim, 2006; Souza, 2004).

Os estudos com a finalidade de extrair, isolar e determinar a estrutura química de princípios ativos presentes nas plantas medicinais iniciaram por volta do século XVIII e XIX, com os químicos (Calixto e Siqueira Junior, 2008; Pint et al., 2002). Desde então são inúmeros os estudos avaliando a atividade farmacológica de derivados de plantas que são utilizadas pela população, assim como acerca da identificação e quantificação dos constituintes químicos responsáveis por esta atividade, auxiliando na comprovação de sua segurança e eficácia (Harvey, 2008).

O delineamento experimental de um projeto envolvendo a pesquisa de plantas medicinais normalmente iniciase através de levantamentos etnobotânicos e/ou etnofarmacológicos e de atividades farmacológicas estudadas por outros pesquisadores já publicadas na literatura. Nos casos em que estas informações são restritas ou ausentes, pesquisadores tem realizado testes farmacológicos preliminares (teste hipocrático), descrito inicialmente por Malone (1983) e adaptado por cada grupo de pesquisadores. Apesar desta ser uma técnica descrita a vários anos, ela tem sido utilizada atualmente por se tratar de uma metodologia simples, o qual é realizado com poucos animais por grupo, e permitem obter resultados relevantes na seleção de dose, via de administração, tempo de latência e efeitos comportamentais induzidos (tóxicos e terapêuticos).

A utilização de animais de laboratório para a avaliação de atividade farmacológica e tóxica tem sido um ponto crucial na pesquisa envolvendo produtos naturais, sendo restritas as metodologias que podem ser substituidas por metodologias in vitro. Porém o uso indiscriminado de animais para esta finalidade representa um dos dilemas mais conflitantes em debates bioéticos (Damy et al., 2010).

Russel e Burch, em 1959, ao elaborarem o Princípio dos $3 \mathrm{Rs}$, propõem aos pesquisadores que seja reduzido o número de animais utilizados em cada experimento, bem como o refinamento das técnicas utilizadas, com o objetivo de evitar a dor e o sofrimento descenessários aos animais. Além disso, os autores propõem que, sempre que possível, seja substituído os experimentos animais por métodos alternativos. A partir de então, diversos grupos de pesquisa têm investido no desenvolvimento de metodologias que permitam a reutilização de animais, redução no número de animais por grupo de tratamento e controle de variáveis ambientais sem comprometer a confiabilidade dos resultados (Damy et al., 2010).

Muitas plantas nativas utilizadas pela população para o tratamento de suas enfermidades apresentam informações científicas restritas quanto a segurança e eficácia dos mesmos, dificultando o direcionamento das pesquisas de forma racional pela maioria dos pesquisadores. Este é o 
caso da da espécie vegetal estudada no presente trabalho, a Tynanthus micranthus Corr. Méllo ex K. Schum. Ela é conhecida popularmente como cipócravo, devido ao forte odor de cravo que exala dos caules, quando cortados ou macerados e é facilmente encontrada em remanescentes florestais na região Noroeste do Estado do Paraná, Brasil (Cansian, 2010; Lopes et al., 2008; Souza et al., 2010).

As espécies do gênero Tynanthus são utilizadas popularmente para diversas finalidades medicinais, e poucas foram amplamente estudas cientificamente. As espécies Tynanthus fasciculatus Miers e Tynanthus elegantus Miers são utilizadas como afrodisíaco, carminativo, estimulante, tônico, para problemas estomacais e para dispepsia (Brandão et al., 2009). A Tynanthus micranthus, em particular, é utilizada popularmente como estimulante e afrodisíaca (Cansian, 2010), porém na região Oeste do Paraná esta planta é utilizada pela população como calmante e analgésica (Bolson, 2009).

Em um levantamento bibliográfico referente à espécie em estudo, observou-se a ausência de trabalhos que comprovem a sua atividade farmacológica de acordo com a indicação popular. Pelo fato da utilização popular indicar um possível efeito relacionado ao Sistema Nervoso Central, mesmo que contraditório (calmante e estimulante), o objetivo do presente trabalho foi avaliar a atividade farmacológica preliminar do extrato aquoso das folhas secas de Tynanthus micranthus, usando o teste hipocrático em camundongos, como uma ferramenta para direcionar estudos farmacológicos futuros, bem como avaliar os custos envolvidos na execução deste estudo.

\section{MATERIAL E MÉTODOS}

\section{Material botânico}

As folhas da Tynanthus micranthus Corr. Méllo ex K. Schum foram coletadas em uma propriedade particular localizada na Linha Jacutinga, município de Vera Cruz do Oeste Paraná, em março de 2010 (entre as 9 e 10 horas). O material botânico foi autenticado pela bióloga M.Sc. Jaqueline Durigon. Uma amostra do mesmo está depositada no Museu Botânico Municipal (MBM) de CuritibaPR, sob o número 231.071.

\section{Análise fitoquímica preliminar}

As folhas secas de $T$. micranthus foram submetidas a reações colorimétricas e de precipitação para a caracterização fitoquímica preliminar (Costa, 2000; Simões et al., 2007), conforme representado na Tabela 1.

\begin{tabular}{|c|c|}
\hline Grupo investigado & Reações \\
\hline Cumarinas voláteis & Reação com hidróxido de sódio e aquecimento \\
\hline Polifenóis gerais & $\begin{array}{l}\text { Reação de precipitação com cloreto férrico e com } \\
\text { acetato de chumbo }\end{array}$ \\
\hline Taninos & Reação de precipitação com gelatina e com alcalóides \\
\hline Catequinas & Reação com ácido clorídrico e aquecimento \\
\hline Flavonóides & $\begin{array}{l}\text { Reação de Cianidina/Shinoda, de Oxalo-Bórica, com } \\
\text { hidróxido de sódio e com cloreto férico }\end{array}$ \\
\hline $\begin{array}{l}\text { Heterosídio } \\
\text { antociânicos }\end{array}$ & Reação com ácido fórmico e aquecimento \\
\hline Antraquinonas & $\begin{array}{l}\text { Reação com hidróxido de sódio, œm ácido clorídrico, } \\
\text { e com hidróxido de amômio em solução toluênica }\end{array}$ \\
\hline Alcalóides & $\begin{array}{l}\text { Reação com reagente de Dragendorff, com reagente } \\
\text { de Mayer e com reagente de Bertrand }\end{array}$ \\
\hline Metilxantinas & $\begin{array}{l}\text { Reação com ácido clorídrico, peróxido de hidrogênio e } \\
\text { hidróxido de amônio (ensaio de murexido) }\end{array}$ \\
\hline Saponinas & $\begin{array}{l}\text { Reação de Liebermann-Buchard, de Salkowski e } \\
\text { Presença e permanência de espuma }\end{array}$ \\
\hline
\end{tabular}

\section{Obtenção do extrato aquoso (EA)}

As folhas maduras $e$ jovens íntegras de $T$. micranthus foram secas em temperatura ambiente por 30 dias e posteriormente trituradas, embalada e armazenada em local apropriado até a sua utilização. $O$ extrato aquoso de $T$. micranthus foi obtido por infusão das folhas secas trituradas em água $(7 \%$ $\mathrm{p} / \mathrm{p}$ ), o qual foi filtrado, liofilizado e posteriormente armazenado à $-20^{\circ} \mathrm{C}$ até a sua utilização. 
Fitoquímica e atividade farmacológica preliminar do extrato aquoso de Tynanthus micranthus corr. Méllo ex k. Schum: um exemplo da capacidade preditiva e dos custos no desenvolvimento de novos produtos farmacêuticos

\section{Animais}

Para este estudo foram utilizados camundongos Swiss machos, com idade entre 3 e 4 meses (pesando entre 35 e $50 \mathrm{~g}$ ), provenientes do Biotério Central da Universidade Federal de Santa Catarina (UFSC). Os animais foram alojados em grupos e mantidos em condições controladas de temperatura $\left(22 \pm 2^{\circ} \mathrm{C}\right)$ em estante ventilada com exaustão Alesco®, ciclo claro/escuro 12/12 h com acesso a água e ração ad libitum. Os animais foram mantidos no laboratório para adaptação por um período de uma hora antes do início dos experimentos. Os protocolos experimentais foram aprovados pelo Comitê de Ética no Uso de Animais da Universidade Federal de Santa Catarina.

\section{Teste hipocrático}

O teste hipocrático foi realizado conforme descrito por Malone (1983), no qual foram utilizados 18 animais, divididos em grupos de acordo com tratamento recebido, conforme ilustrado na Tabela 2.

Tabela 2 - Representação dos grupos, tratamentos, doses e vias de
administração dos animais avaliados no teste hipocrático.

Os animais foram tratados e observados $30 \mathrm{~min}, 1 \mathrm{~h}, 2 \mathrm{~h}, 3 \mathrm{~h}, 24 \mathrm{~h}$ após a administração oral do extrato e, a partir de então, diariamente até 0 décimo dia, uma vez ao dia. Foram avaliados os efeitos relacionados à atividade no Sistema Nervoso Central (estimulante, depressora, entre outros), Sistema Nervoso Autônomo (salivação, diarréia, entre outros) e a mortalidade dos animais tratados quando comparados com os animais controles. Os efeitos observados foram registrados em um protocolo adaptado daquele proposto por Malone (1983), respeitando uma escala de intensidade dos efeitos, sendo (0) sem efeito, (-) efeito diminuído, $(+)$ efeito presente e $(++)$ efeito intenso, para posteriormente serem analisados.

\section{Cálculo do custo contábil}

O cálculo do custo contábil foi realizado para simular o valor gasto por uma empresa privada na realização destas atividades de Pesquisa e Desenvolvimento, considerando que a mesma apresente uma estrutura apropriada para esta finalidade (estrutura física, equipamentos, reagentes e funcionários qualificados). Sendo assim, todas as atividades realizadas, inclusive a de manutenção do biotério e do extrato liofilizado em freezer, foram divididas em etapas para facilitar a apresentação dos resultados, conforme ilustrado na Figura 1.

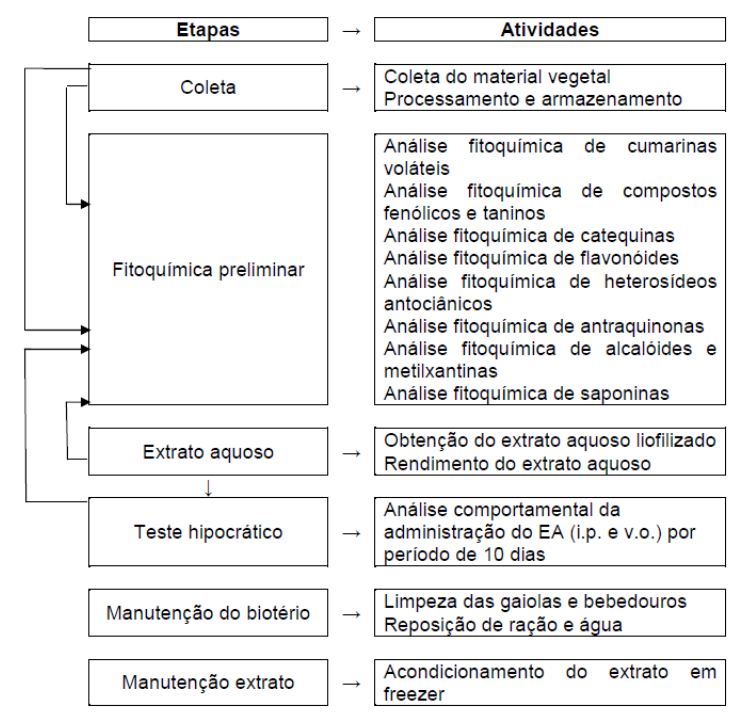

Figura 1 - Representação das etapas e atividades realizadas durante um estudo de triagem farmacológica com um extrato vegetal.

O custo total das atividades executadas (Custo total) foi calculado utilizando os conceitos básicos de administração de empresas, o qual leva em consideração os custos quanto a mão-de-obra direta necessária para a realização de todas as atividades, 
material utilizado para as mesmas e depreciação contábil de todos os equipamentos (Sebrae, 2006). De modo geral, o custo total foi calculado utilizando a fórmula abaixo:

Custo total $=$ Custo mão-de-obra direta + Custo material aplicado + Depreciação contábil

O cálculo dos custos de mão-deobra, material aplicado e depreciação contábil foram realizados levando em consideração o tempo necessário para a execução de cada etapa (Sebrae, 2006). Os itens contabilizados em cada uma delas estão descritas na Tabela 3. Os resultados foram expressos em valores monetários (real - $\mathrm{R} \$$ ) e porcentagem calculados para cada etapa.

\begin{tabular}{|c|c|c|c|}
\hline Etapa & $\begin{array}{l}\text { Custo de mão- } \\
\text { de-obra direta }\end{array}$ & $\begin{array}{c}\text { Custo do material } \\
\text { aplicado }\end{array}$ & $\begin{array}{c}\text { Depreciação } \\
\text { contábil }\end{array}$ \\
\hline Coleta & $\begin{array}{l}\text { Viagem, coleta, } \\
\text { classificação e } \\
\text { processamento }\end{array}$ & $\begin{array}{l}\text { Combustivel e } \\
\text { material de escritório } \\
\text { para identificação }\end{array}$ & $\begin{array}{l}\text { Veículo, } \\
\text { utensílios para } \\
\text { coleta e } \\
\text { equipamentos } \\
\text { para o } \\
\text { processamento }\end{array}$ \\
\hline $\begin{array}{l}\text { Fitoquímica } \\
\text { preliminar }\end{array}$ & $\begin{array}{l}\text { Preparo das } \\
\text { soluções, } \\
\text { realização das } \\
\text { análises e } \\
\text { avaliação dos } \\
\text { resultados }\end{array}$ & Reagentes & Equipamentos \\
\hline Extrato aquoso & $\begin{array}{l}\text { Preparo, } \\
\text { processamento, } \\
\text { armazenamento } \\
\text { e avaliação dos } \\
\text { resultados }\end{array}$ & Filtros & Equipamentos \\
\hline Teste hipocrático & $\begin{array}{l}\text { Preparo das } \\
\text { soluções, } \\
\text { experimentos e } \\
\text { avaliação dos } \\
\text { resultados }\end{array}$ & $\begin{array}{l}\text { Seringas, agulhas e } \\
\text { animais }\end{array}$ & $\begin{array}{l}\text { Cânulas de } \\
\text { gavagem e } \\
\text { equipamentos }\end{array}$ \\
\hline $\begin{array}{l}\text { Manutenção } \\
\text { Biotério }\end{array}$ & $\begin{array}{l}\text { Limpeza e } \\
\text { manutenção }\end{array}$ & Serragem e ração & $\begin{array}{l}\text { Estante ventilada } \\
\text { para } \\
\text { camundongos, } \\
\text { caixas e } \\
\text { bebedouros }\end{array}$ \\
\hline $\begin{array}{l}\text { Manutenção } \\
\text { Extrato }\end{array}$ & Armazenamento & - & Freezer \\
\hline
\end{tabular}

\section{RESULTADOS}

\section{Análise fitoquímica preliminar}

A Tabela 4 demonstra os resultados para os testes realizados na avaliação fitoquímica preliminar de grupos químicos de metabólitos encontrados nas folhas da planta Tynanthus micranthus. Como pode ser observado, as reações foram positivas para cumarinas voláteis, polifenóis, taninos, catequinas, flavonóides e saponinas.

Para flavonóides, observou-se que os resultados obtidos nas diferentes reações indicam a presença de flavona, semelhante ao resultado encontrado por Cansian (2010) em frações clorofórmio, acetato de etila e hidroalcoólica na raiz, caule e folhas da planta $T$. micranthus. Enquanto que para saponinas, ocorreu reação positiva para a Reação de Salkowski, indicando a presença e núcleo esteroidal.

Tabela 4 - Avaliação fitoquímica preliminar das folhas de Tynanthus micranthus.

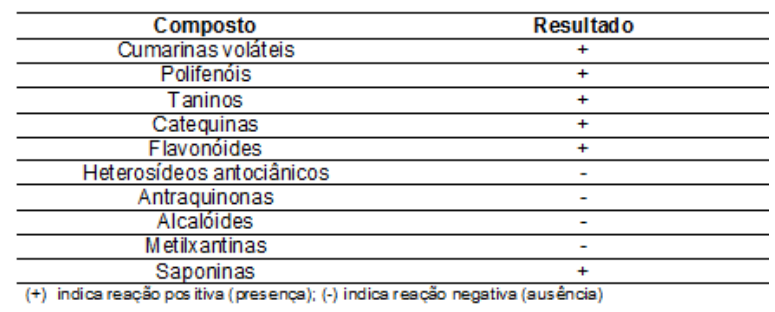

\section{Teste hipocrático}

Os principais efeitos encontrados nos animais tratados com EA de $T$. micranthus, nas doses de $1000 \mathrm{mg} / \mathrm{kg}$ e 2000 mg/kg (v.o. e i.p.), quando comparados ao grupo controle, foram observados nos tempos de $30 \mathrm{~min}, 1 \mathrm{~h}$, $2 \mathrm{~h}$ e $3 \mathrm{~h}$, sendo estes apresentados na Tabela 5 e 6 . A partir deste tempo, não foram observados efeitos significativos, quando comparados com o grupo controle.

Dentre os efeitos observados, destacam-se a analgesia (30 min a $3 \mathrm{~h}$ ), a sedação (30 min a $2 \mathrm{~h}$, em grupos tratados por via i.p.) e a piloereção (30 $\min a 1 \mathrm{~h}$ ) devido à intensidade $\mathrm{e}$ duração do mesmo, bem como a concentração e a via de administração do extrato. Além disso, não houve mortalidade dos animais observados, o que indica uma baixa toxicidade aguda desta planta. 
Fitoquímica e atividade farmacológica preliminar do extrato aquoso de Tynanthus micranthus corr. Méllo ex k. Schum: um exemplo da capacidade preditiva e dos custos no desenvolvimento de novos produtos farmacêuticos

Tabela 5 - Principais efeitos observados, no teșe hipocrático, em camundongos Swiss após tratamento agudo, por via oral, con EA das folhas de Tynanthus micranthus.

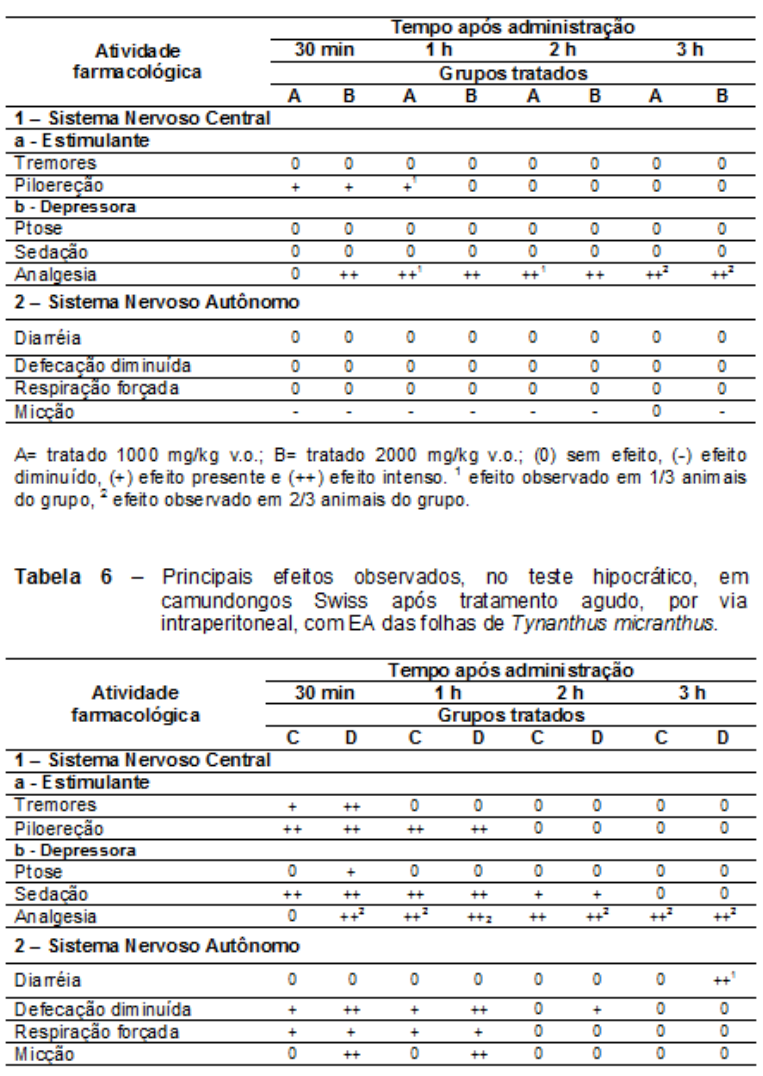

$\mathrm{C}=1000 \mathrm{mg} / \mathrm{kg}$ i.p.; $\mathrm{D}=2000 \mathrm{mg} / \mathrm{kg}$ i.p.; (0) sem efeito, $(-)$ efeito diminuido, $(+)$ efeito presente $\mathrm{e}(++)$ efeito intenso. ${ }^{2}$ efeito observado em $2 / 3$ an imais do grupo.

\section{Cálculo do custo contábil}

Conforme apresentado na Tabela 7, o presente estudo apresentou um custo total de $R \$ 1610,20$, sendo os custos de mão-de-obra direta na etapa de coleta $(R \$ 299,46)$ e na manutenção do biotério $(R \$ 283,36)$ os valores mais altos.

Quando analisado o valor total de cada uma das etapas realizadas, observou-se que a manutenção do biotério foi o que apresentou maior custo, representando 39\% dos custos totais, enquanto que a etapa de manutenção do extrato foi a que apresentou menor custo (7\%). Além disso, os dados apresentados na Figura 2 mostram a importância do custo de mão-de-obra direta, uma vez que ele representa $59 \%$ do custo total do estudo. É importante ressaltar que entre os custos apresentados não foram inclusos os custos com mão-de-obra especializada para a criação dos animais até o momento de seu uso, assim como os custos estruturais e administrativos do biotério (luz, água, vacinas, tratamentos antiparasitários, desgaste dos equipamentos de refrigeração da sala do biotério central, entre outros).

\begin{tabular}{|c|c|c|c|c|}
\hline Etapa & $\begin{array}{c}\text { Custo mão- } \\
\text { de-ob ra } \\
\text { direta }\end{array}$ & $\begin{array}{l}\text { Custo material } \\
\text { aplicado }\end{array}$ & $\begin{array}{c}\text { Dep reciação } \\
\text { contábil }\end{array}$ & Total \\
\hline Coleta & $\mathrm{R} \$ 299,46$ & $\mathrm{R} \$ 81,91$ & $\mathrm{R} \$ 0,33$ & $\mathrm{R} \$ 381,70$ \\
\hline \multicolumn{5}{|l|}{ Fitoquímica } \\
\hline preliminar & $R \$ 155,53$ & $\mathrm{R} \$ 4,74$ & $\mathrm{R} \$ 2,30$ & $\mathrm{R} \$ 162,57$ \\
\hline Extrato aquoso & $\mathrm{R} \$ 138,46$ & $\mathrm{R} \$ 0,15$ & R\$ 17,88 & $\mathrm{R} \$ 156,49$ \\
\hline Teste hipocrático & $\mathrm{RS} 69,23$ & $\mathrm{R} \$ 100,93$ & $\mathrm{RS} 0,02$ & $\mathrm{R} \$ 170,18$ \\
\hline \multicolumn{5}{|l|}{ Manutenção } \\
\hline Extrato & $\mathrm{R} \$ 1,61$ & $\mathrm{R} \$ 0,00$ & $R \$ 110,96$ & $\mathrm{R} \$ 112,57$ \\
\hline $\begin{array}{l}\text { Manutenção } \\
\text { Biotério }\end{array}$ & $\mathrm{R} \$ 283,36$ & $\mathrm{R} \$ 67,17$ & $\mathrm{R} \$ 276,16$ & $\mathrm{R} \$ 626,69$ \\
\hline Total & R\$ 947,65 & $\mathrm{R} \$ 254,90$ & $\mathrm{R} \$ 407,65$ & $R \$ 1610,20$ \\
\hline
\end{tabular}
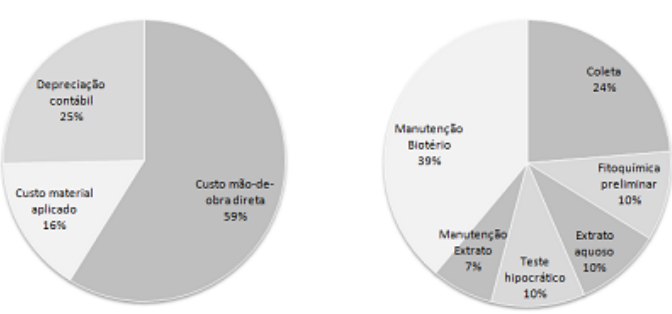

Figura 2 - Representação, em porcentagem, o peso de cada categoria (esquerda) de custo e de cada etapa (direita) no valor de custo total das atividades realizadas

\section{DISCUSSÃO}

A pesquisa fitoquímica é uma ferramenta importante quando se deseja conhecer os constituintes químicos de espécies vegetais ou avaliar a sua presença. Em casos onde não se tem estudos químicos sobre a espécie de interesse ou quando estes são restritos, a análise fitoquímica preliminar pode indicar a presença de grupos de metabólitos secundários relevantes na mesma. Porém, quando o interesse é restrito a uma classe específica de constituintes ou a alguns compostos isolados, a investigação deverá ser direcionada para $o$ isolamento e a elucidação estrutural das mesmas (Simões et al., 2007). 
Para a espécie Tynanthus micranthus, a quantidade disponível de informações referentes à análise fitoquímica é restrita. No trabalho realizado por Cansian (2010), a presença de grupos de metabólitos secundários foi avaliada em diferentes extratos $(H$ - hexano, $C$ - clorofórmio, $A E$ - acetato de etila e HA - hidroalcoólica) de raiz $(R)$, caule $(C)$ e folha $(F)$ da espécie vegetal Tynanthus micranthus Corr. Méllo ex K. Schum. O trabalho demonstra a presença de leucoantocianidinas nos extratos $\mathrm{CR}$, AER, HAR e HAF; heterosídeos flavônicos nos extratos CR, AER, HAR, CC, AEC, HAC, CF, AEF e HAF; heterosídeos antraquinônicos nos extratos HAC e HAF, e esteróides e/ou tripertenos nos extratos $\mathrm{HR}, \mathrm{CR}, \mathrm{HC}$, CC e HF.

Este estudo revelou a presença de alguns compostos isolados, entre eles um hidrocarboneto de cadeia longa, com estrutura não completamente elucidada, a flavona apigenina e o esteróide $\beta$-sitosterol. No óleo volátil extraído das folhas de Tynanthus micranthus foram identificados os compostos O-decil-hidroxilamina, hexadecametil-ciclooctasiloxane, éster butil, éster com butil glicolato, metilhexadecanoato, ácido 1,2 benzenodicarboxílico, butil 2-etilexil éster, ácido hexadecanóico (ácido palmítico), pentadecanal, ciclodecasiloxane, eicosametil, 5dodeciloxolan-2-ona e ácido oléico (Cansian, 2010).

A análise fitoquímica preliminar realizada em nosso trabalho mostrou a presença de cumarinas voláteis, polifenóis, taninos, catequinas, flavonóides e saponinas nas folhas da planta Tynanthus micranthus Corr. Méllo ex K. Schum. A presença de destes grupos de metabólitos secundários foram confirmados pela literatura como compostos presentes em diversas espécies deste gênero. A única exceção é para as catequinas, onde não se encontrou relatos da presença deste grupo neste gênero.

Esta variação nos resultados quanto à presença de grupos de metabólitos secundários pode ser relacionada com os diversos fatores que interferem na quantidade e até mesmo na natureza dos constituintes ativos. Os fatores que influenciam na produção de metabólitos secundários pelas espécies vegetais são: sazonalidade, ritmo circadiano, desenvolvimento, temperatura, disponibilidade hídrica, radiação ultravioleta, nutrientes (macromoléculas e micromoléculas), poluição atmosférica, altitude e indução por estímulos mecânicos ou ataque de patógenos (Gobbo-Neto e Lopes, 2007; Simões et al., 2007).

Quanto à avaliação da atividade farmacológica, o teste hipocrático é utilizado como uma ferramenta para direcionar os estudos farmacológicos, principalmente quando se tem pouco conhecimento sobre características farmacocinéticas e farmacodinâmicas da substância a ser testada, como é o caso da espécie $T$. micranthus, cujas informações científicas são ainda mais restritas. Os seus resultados auxiliam na seleção de doses, via de administração, tempo de latência e efeitos comportamentais induzidos, além de representar um teste preliminar de toxicidade aguda.

Dentre os efeitos observados nos animais tratados com 0 EA de $T$. micranthus, é importante ressaltar o seu efeito analgésico e a sua baixa toxicidade, uma vez que não houve mortalidade no tratamento agudo dos animais com doses altas (até $2 \mathrm{~g} / \mathrm{kg}$ ). Estes dados indicam que a $T$. micranthus tende a ser uma planta promissora para o desenvolvimento de novos produtos com ação analgésica e 
Fitoquímica e atividade farmacológica preliminar do extrato aquoso de Tynanthus micranthus corr. Méllo ex k. Schum: um exemplo da capacidade preditiva e dos custos no desenvolvimento de novos produtos farmacêuticos

que a probabilidade de se obter resultados satisfatórios é alta.

Estudos realizados por Custódio e colaboradores (2010) indicam a presença de majoritária de eugenol no óleo essencial de folhas de $T$. micranthus, extraído por hidrodestilação, sendo 0 efeito analgésico e o mecanismo de ação desse composto aplamente conhecido (Markowitz et al., 1992; Pramod et al., 2010).

O efeito analgésico observado nos animais tratados de forma aguda com o EA no teste hipocrático traz indícios da presença de eugenol nesta forma de extração, sendo, no entanto, necessário a realização de testes fitoquímicos apropriados para comprovar esta hipótese. A investigação fitoquímica dos compostos presentes no EA de $T$. micranthus permitirá um maior conhecimento sobre os efeitos esperados do mesmo na administração em animais, direcionando o projeto de pesquisa de modo racional, aumentando a probabilidade de sucesso e reduzindo os custos envolvidos na realização deste.

Por outro lado, a avaliação de custo de um estudo é uma prática comum na maioria dos centros de P\&D, onde a avaliação de risco, retorno financeiro e viabilidade do mesmo são critérios fundamentais para a sua aprovação e posterior execução. Todos os projetos de P\&D convivem com um componente de incerteza com relação aos seus resultados, porém quanto maior o desconhecimento com relação aos resultados esperados, maior o risco relacionado ao projeto. Sendo assim, a avaliação criteriosa de projetos e dos resultados apresentados ao final de cada etapa tem auxiliado gestores de empresas farmacêuticas a decidirem por sua continuidade ou não, bem como os investimentos a serem realizados (Pinheiro et al., 2006; Valeriano, 1998).
No meio acadêmico, a preocupação quanto aos custos relacionados com a execução de um trabalho, na maioria das vezes, dizem respeito à compra de equipamentos $e$ reagentes necessários, não levando em conta os custos com mão-de-obra direta. Desta forma, o presente trabalho demonstra uma maneira simples e prática de calcular os principais custos relacionados com a execução de um projeto, baseada em conhecimentos básicos amplamente difundidos no que se refere a administração de empresas, porém adaptada para a rotina de um laboratório de P\&D e relatando a importância que o custo quanto a mãode-obra direta qualificada representa no valor final do projeto.

Os resultados demonstram que, com um custo direto de $R \$ 1610,20$, a espécie vegetal em questão indica resultados positivos em testes que envolvam a avaliação de efeitos analgésicos. Dentre as etapas de atividades realizadas, o teste hipocrático e a análise fitoquímica preliminar obtiveram um custo total de $\mathrm{R} \$ 170,18 \mathrm{e}$ $R \$ 162,57$, respectivamente, sendo que cada um representa aproximadamente $10 \%$ do custo total do estudo.

Além do teste hipocrático ser um teste de baixo custo, ele proporciona resultados importantes na decisão da via de administração, toxicidade aguda, efeitos com altas doses e diferentes vias, além do tempo de latência para os efeitos observados. Com essas informações, e uma análise fitoquímica mais aprofundada, isolando e identificando os compostos majoritários, é possível avaliar na literatura a relação composto $x$ atividade farmacológica direcionando de forma mais assertiva as pesquisas a serem realizadas.

Desta forma, nosso estudo demonstra aos pesquisadores de diferentes áreas uma maneira simples de calcular os custos envolvidos com 
um experimento de triagem inicial para plantas ditas medicinais, seja após a sua execução (por meio de dados reais) ou na forma de uma estimativa (por meio de dados teóricos).

Além disso, apresentamos dados inéditos (a atividade analgésica da $T$. micranthus), mostrando uma estratégia de triagem farmacológica simples e de baixo custo para a pesquisa de plantas medicinais nativas que apresentem informações restritas na literatura.

\section{CONCLUSÃO}

Os resultados de nosso estudo indicam que a espécie Tynanthus micranthus tende a ser uma planta promissora para o desenvolvimento de novos produtos com ação analgésica e com baixa toxicidade aguda.

A avaliação dos custos relacionados a realização deste projeto nos levam a concluir que a avaliação farmacológica preliminar, pelo teste hipocrático em camundongos, mostra resultados relevantes para 0 direcionamento da pesquisa. Por se tratar de um teste relativamente simples e de baixo custo, pode ser considerado como uma ferramenta para direcionar os estudos farmacológicos, principalmente quando se tem pouco conhecimento sobre características farmacocinéticas e farmacodinâmicas da substância a ser testada.

\section{AGRADECIMENTOS}

Agradecemos aos Drs. Luiz e Carmen Donaduzzi, do Lab. Farmacêutico Prati-Donaduzzi, e à Dra. Josélia Lager Manfio, da BIOCINESE Centro de Estudos Farmacêuticos, pelo apoio financeiro e logístico; à UNIPAR, na pessoa de Euclides Lara Cardozo Junior pelo apoio institucional; e aos professores da UFSC participantes do
Mestrado Profissional em parceria com a UNIPAR.

\section{NOTAS INFORMATIVAS}

Protocolo experimental aprovado pelo Comitê de Ética Sobre o Uso de Animais da Universidade Federal de Santa Catarina número 23080.040914/2010-46/CEUA/UFSC.

\section{REFERÊNCIAS}

BOLSON, M. Levantamento etnobotânico de plantas medicinais nativas utilizadas pela população dos municípios de São Pedro do Iguaçu e Vera Cruz do Oeste, Paraná, Brasil. 2009. Toledo, 62 f. Trabalho de Conclusão de Curso (Curso de Bacharelado em Ciências Biológicas) - Pontifícia Universidade Católica do Paraná.

BRANDÃO, M.G.L. et al. Traditional uses of American plant species from the 1st edition of Brazilian Official Pharmacopoeia. Revista Brasileira de Farmacognosia, v.19, n.2a, p.478-487, 2009.

CALIXTO, J.B.; SIQUEIRA JUNIOR, J.M. Desenvolvimento de medicamentos no Brasil: Desafios. Gazeta Médica da Bahia, v.78, n.1, p.98-106, 2008.

CANSIAN, F.C. Estudo fitoquímico e atividades biológicas da espécie Tynanthus micranthus Corr. Méllo (Bignoniaceae). 2010. Curitiba, 101 f. Dissertação (Mestrado em Ciências Farmacêuticas) - Programa de Pós Graduação em Ciências Farmacêuticas, Universidade Federal do Paraná.

COSTA, A.F. Farmacognosia. Farmacognosia Experimental. Lisboa: Fundação Calouste Gulbenkian, 2000. 992 p.

CUSTÓDIO, D.L. et al. Antimicrobial activity of essential oils from Pimenta pseudocaryophyllus and Tynanthus micranthus. Brazilian Archives of Biology and Technology, v.53, n.6, p.13631369, 2010.

DAMY, S.B. et al. Aspectos fundamentais da experimentação animal - aplicações em cirurgia experimental. Revista da Associação Médica Brasileira, v.56, n.1, p.103-111, 2010.

GOBBO-NETO, L.; LOPES, N.P. Plantas medicinais: fatores de influência no conteúdo de metabólitos secundários. Química Nova, v.30, n.2, p.374-381, 2007. 
GURIB-FAKIM, A. Medicinal plants: Traditions of yesterday and drugs of tomorrow. Molecular Aspects of Medicine, v.27, n.1, p.1-93, 2006.

HARVEY, A.L. Natural products in drug discovery. Drug Discovery Today, v.13, n.1920, p.894-901, 2008.

LOPES, M.M.M. et al. Crescimento de mudas de cipó-cravo (Tynanthus fasciculatus Miers), uma liana com potencial medicinal. Revista Árvore, v.32, n.2, p.211-216, 2008.

MALONE, M.H. The pharmacological evaluation of natural products - general and specific approaches to screening ethnopharmaceuticals. Journal of Ethnopharmacology, v.8, n.2, p.127-147, 1983.

MARKOWITZ, K. et al. Biologic properties of eugenol and zinc oxide-eugenol: A clinically oriented review. Oral Surgery, Oral Medicine, Oral Pathology, v.73, n.6, p.729-737, 1992.

PINHEIRO, A.A. et al. Metodologia para gerenciar projetos de pesquisa $e$ desenvolvimento com foco em produtos: uma proposta. Revista de Administração Pública, v.40, n.3, p.457-478, 2006.

PINTO, A.C. et al. Produtos naturais: atualidade, desafios e perspectivas. Química Nova, v.25, n.1, p.45-61, 2002.

PRAMOD, K.; ANSARI, S.H.; ALI, J. Eugenol: a natural compound with versatile pharmacological actions. Natural Product Communications, v.5, n.12, p.1999-2006, 2010.

SEBRAE, Custos na prestação de serviços, Série Saiba Mais - SEBRAE, 2006. Disponível em:

$<$ http://www.biblioteca.sebrae.com.br/bds/bds.ns f/B83E6E16A0097D3A03257146005A1566/\$File /NT000AFA42.pdf>. Acesso em: 13 abr. 2011.

SIMÕES, C.M.O. et al. Farmacognosia: da planta ao medicamento. Porto Alegre: Editora da URGS, 2007. 1104 p.

SOUZA, L.A.D.; SANTOS, G.D.O.; MOSCHETA, I.S. Morfoanatomia floral de espécies lianescentes de Bignoniaceae. IHERINGIA, v.65, n.1, p.5-15, 2010.

SOUZA, M.V.N.D. Novos produtos naturais capazes de atuar na estabilização de microtúbulos, um importante alvo no combate ao câncer. Química Nova, v.27, n.2, p.308-312, 2004.

VALERIANO, D.L. Gerência em projetos de pesquisa, desenvolvimento e engenharia. São Paulo: Makron Books, 1998. 466 p. 